\title{
14. Induction of Ornithine Decarboxylase Activity in Mouse Skin by a Possible Tumor Promoter, Okadaic Acid*)
}

\author{
By Hirota FujIKI,**) Masami Suganuma,**) Hiroko SugurI,**) \\ Shigeru Yoshizawa,**) Makoto OJIKa,***) Kazumasa WaKamatsu,***; \\ Kiyoyuki YamadA,***) and Takashi Sugimura, M. J. A.****) \\ (Communicated Feb. 12, 1987)
}

Okadaic acid is a polyether derivative of a $\mathrm{C}_{38}$ fatty acid, which was first isolated independently from two sponges, Halichondria okadai, a black sponge, by Scheuer's group and Halichondria melanodocia by Schmitz's group (Tachibana et al., 1981), and was subsequently isolated from a dinoflagellate, Prorocentrum lima by Yasumoto's group (Murakami et al., 1982) (Fig. 1). Okadaic acid is believed to be a metabolite of an epiphytic microorganism. 35-Methylokadaic acid, named dinophysistoxin-1, was isolated from the hepatopancreas of the mussel Mytilus edulis, as a causative agent of diarrhetic shellfish poisoning in Japan (Murata et al., 1982). Okadaic acid was also found in European mussels as the toxic principle of a seafood disease (Kumagai et al., 1986).

Since okadaic acid is known to cause damage in the epithelium of the gastrointestinal tract after its administration to mice, we examined it by the irritant test on mouse ear, which is a screening test for tumor promoters. Okadaic acid was isolated from the black sponge, Halichondria okadai, obtained along the coast near Anori and Koga, Mie Prefecture in Japan. Application of $80 \mathrm{ng}$ of okadaic acid to the ears of mice induced redness. This potency was about one-tenth of that of the tumor promoter teleocidin.

Our short-term screening system for tumor promoters consists of three successive tests; namely, tests of irritation of mouse ear and inductions of ornithine decarboxylase (ODC) in mouse skin and of HL-60 cell adhesion (Fujiki and Sugimura, 1987). Since okadaic acid induced a positive response in the irritant test, we next tested its ability to induce ODC in mouse skin.

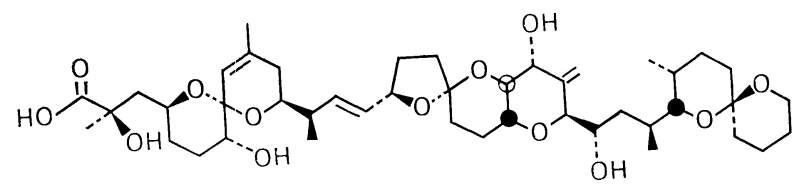

Fig. 1. Structure of okadaic acid.

*) This work was supported in part by Grants-in-Aid for Cancer Research from the Ministry of Education, Science and Culture, and from the Ministry of Health and Welfare for a Comprehensive 10-Year Strategy of Cancer Control, Japan, and by a grant from the Princess Takamatsu Cancer Research Fund.

**) Cancer Prevention Division, National Cancer Center Research Institute, 5-1-1 Tsukiji, Chuo-ku, Tokyo 104.

***) Department of Chemistry, Faculty of Science, Nagoya University, Chikusa, Nagoya 464.

****) National Cancer Center, 5-1-1 Tsukiji, Chuo-ku, Tokyo 104. 


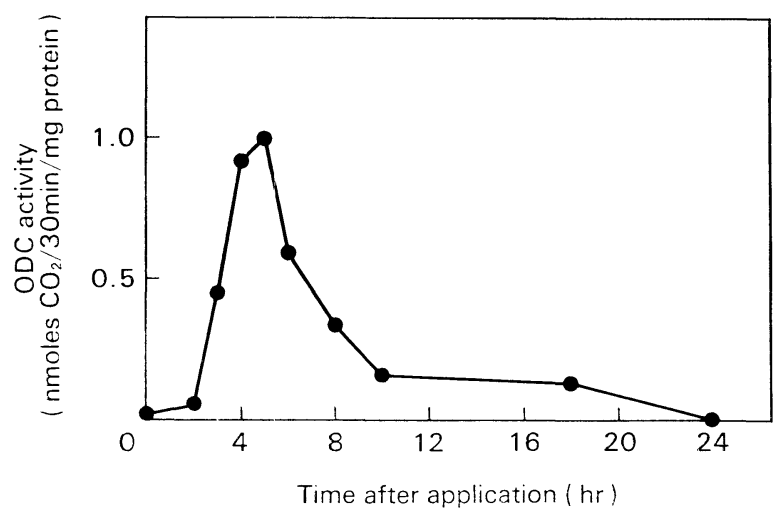

Fig. 2. Time course of induction of ODC activity after application of a solution of $7.5 \mu \mathrm{g}$ of okadaic acid in $0.2 \mathrm{ml}$ of acetone to mouse skin.

Fig. 2 shows the time-course of ODC induction by a single application of $7.5 \mu \mathrm{g}$ of okadaic acid to the skin of the back of 8-week-old female CD-1 mice. The ODC activity increased gradually from $3 \mathrm{hr}$ after application of the compound reaching a maximum in $4-5 \mathrm{hr}$. The time-course of ODC induction by okadaic acid was similar to those of 12-O-tetradecanoylphorbol-13-acetate (TPA) (O'Brien et al., 1975) and teleocidin (Fujiki et al., 1981). In a dose-response experiment application of $7.5-10 \mu \mathrm{g}$ of okadaic acid caused maximal ODC induction.

Pretreatment of the skin with 13-cis-retinoic acid is known to inhibit induction of ODC by TPA and teleocidin (Verma and Boutwell, 1977; Fujiki et al., 1981). Therefore, we examined the effect of 13-cis-retinoic acid on ODC induction by okadaic acid. Pretreatment of the skin with $3.8 \mu \mathrm{g}$ (12.7 nmoles) of 13-cisretinoic acid caused $97.5 \%$ inhibition of ODC induction by $10 \mu \mathrm{g}$ (12.7 nmoles) okadaic acid. These results suggested that okadaic acid, like phorbol ester and teleocidin, has the same response to mouse skin with and without 13-cis-retinoic acid, and may be a tumor promoter in mouse skin.

A derivative of okadaic acid, okadaic acid tetramethyl ether was synthesized from okadaic acid by methylation with methyl iodide and sodium hydride in dimethylformamide followed by hydrolysis with potassium hydroxide in ethanol. Application of $7.5 \mu \mathrm{g}$ of okadaic acid tetramethyl ether did not induce any ODC activity in mouse skin. These results suggest that hydroxyl groups of okadaic acid are apparently necessary for the induction of ODC.

Since ODC induction by okadaic acid was similar to those of phorbol ester and teleocidin, we suppose that okadaic acid may be a tumor promoter like phorbol ester and teleocidin in mouse skin. Recently, we showed that tumor promoters can be classified into TPA-type tumor promoters and non-TPA type tumor promoters, depending on their ability to bind to phorbol ester receptors in cell membranes (Fujiki and Sugimura, 1987). Through further investigation, it should be possible to determine whether or not okadaic acid can bind to phorbol ester receptors.

It is noteworthy that mussels containing okadaic acid and related compounds have caused diarrhetic shellfish poisoning in several countries, such as Japan, Chile, Norway, Holland and Spain, which are known to have high incidences of 
stomach cancer (Waterhouse et al., 1982). Thus the tumor promoting activity of okadaic acid in the skin and also the stomach should be tested.

\section{References}

Fujiki, H. et al. (1981) : Proc. Natl. Acad. Sci. USA., 78, 3872-3876.

Fujiki, H., and Sugimura, T. (1987): Advances in Cancer Res. (in press).

Kumagai, M. et al. (1986): Agric. Biol. Chem., 50, 2853-2857.

Murakami, Y. et al. (1982) : Bull. Japan. Soc. Sci. Fish., 48, 69-72.

Murata, M. et al. (1982) : ibid., 48, 549-552.

O'Brien, T. G. et al. (1975) : Cancer Res., 35, 1662-1670.

Tachibana, K. et al. (1981) : J. Am. Chem. Soc., 103, 2469-2471.

Verma, A. K., and Boutwell, R. K. (1977) : Cancer Res., 37, 2196-2201.

Waterhouse, J. et al. (1982) : Cancer Incidence in Five Countries. vol. IV, IARC Lyon. 